\section{Physical activity during pregnancy and maternal-child health outcomes: a systematic literature review}

\author{
Atividade física na gestação e desfechos da \\ saúde materno-infantil: uma revisão sistemática \\ da literatura
}

Instituto de Nutrição Josué
de Castro, Universidade
Federal do Rio de Janeiro,
Rio de Janeiro, Brasil
2 Instituto de Medicina
Social, Universidade do
Estado do Rio de Janeiro,
Rio de Janeiro, Brasil.
Correspondence
G. Kac
Instituto de Nutrição Josué de
Castro, Universidade Federal
do Rio de Janeiro.
Avenida Brigadeiro
Trompowski s/n, Bloco J,
2o andar, sala 29,
Rio de Janeiro, RJ
21941-590, Brasil.
kacetal@gmail.com

\section{Abstract}

A systematic literature review was conducted to investigate the effects of physical activity during pregnancy on selected maternal-child health outcomes. The search included articles published from 1980 to 2005 in the MEDLINE and LILACS databases using key words such as physical activity, physical exercise, pregnancy, and gestation. The methodological quality of $37 \mathrm{se}$ lected articles was evaluated. It appears to be a consensus that some light-to-moderate physical activity is not a risk factor and may even be considered a protective factor for some outcomes. However, some studies found an association between specific activities (e.g., climbing stairs or standing for long periods) and inadequate birth weight, prematurity, and miscarriage. Few studies found an association between physical activity and maternal weight gain, mode of delivery, or fetal development. Further research is needed to fill these gaps and provide guidelines on the intensity, duration, and frequency of physical activity during pregnancy.

Motor Activity; Exercise; Pregnancy; Review

\author{
Michael Maia Schlüssel 1 \\ Elton Bicalho de Souza 1 \\ Michael Eduardo Reichenheim ${ }^{2}$ \\ Gilberto Kac ${ }^{1}$
}

\section{Introduction}

Evidence from scientific studies points to the important role of physical activity in health promotion and quality of life and the prevention and control of various diseases 1 . However, for individuals in specific physiological conditions, such benefits may not always occur, or are valid with certain restrictions.

Until a few decades ago, pregnant women were advised to reduce their activities and even interrupt their occupational work, especially in the final stage of pregnancy 1 . However, since the 1990s experts have admitted the positive effect of regular physical activity during gestation and have even encouraged it, as long as the woman does not present specific adverse conditions 2 .

In fact, there appears to be a consensus that maintaining light to moderate physical activity during an uncomplicated pregnancy provides various benefits for the woman's health ${ }^{3}$. This is explained by the fact that physical activity causes a thermal response and circulatory redistribution, shifting the blood concentration from the uterus and placenta to the extremities. This process helps reduce and prevent lower back pain, fosters lower liquid retention, reduces cardiovascular stress, increases the oxygenation capacity, decreases blood pressure, reduces the risk of gestational diabetes, prevents thromboses and varicose veins, and helps control gestational weight gain $4,5,6$. 
The advantages also include emotional aspects, since physical activity helps make the pregnant woman more self-confident and satisfied with her appearance, in addition to raising her self-esteem, thus reducing the risks of postpartum depression 1,3 .

However, physical activity during pregnancy still raises some controversy. According to Gallup 7 , the published studies and guidelines on physical activity during pregnancy are insufficient. According to Morris \& Johnson 8, although limited, the literature suggests that practicing moderate exercise during a pregnancy with no additional risks does not lead to undesirable outcomes for either the mother or the fetus. These authors go on to contend that more studies are necessary.

In addition, the benefits of physical activity during pregnancy do not appear to be widely publicized, and some consider the theme a taboo. Many women believe that the physiological limitations posed by pregnancy prevent them from participating in programs that promote regular exercise 9 . Others believe that to remain rested and relaxed during pregnancy is significantly more important than exercising or maintaining an active lifestyle 10 .

Considering the persistent controversies on the theme, the current study aims to conduct a systematic review of the scientific articles on the association between physical activity during pregnancy and the occurrence of maternal-child health outcomes. The review focused specifically on two aspects of pregnant women's physical activity: occupational physical activities and leisure-time physical activities. The former include activities pertaining to the individual's occupation (paid or unpaid), i.e., all activities performed in their place of work, including housework. Leisure-time physical activities include activities performed with the purpose of health promotion or simply for leisure.

\section{Methods}

The search was conducted in July 2006, and the articles were identified through a literature search of the MEDLINE and LILACS databases, using the following key words: (physical activity OR physical exercise) AND (pregnancy OR gestation). As the inclusion criteria, the articles had to have been published from 1980 to 2005, in Portuguese, English, or Spanish, and refer to crosssectional, case-control, or follow-up (cohort) epidemiological studies. The principal outcomes of the studies had to be preeclampsia; gestational arterial hypertension; gestational diabetes mel- litus; gestational weight gain; miscarriage; mode of delivery; fetal growth or development; birth weight; length at birth; or prematurity.

The search process initially involved a survey of the references based on the key words, which located 3,313 articles. These references were then sifted using some of the inclusion criteria as search filters: articles with an available abstract $(2,703)$; published from 1980 to $2005(2,488)$; with a sample consisting of humans $(1,269)$; females $(1,178)$; and published in English, Portuguese, or Spanish $(1,085)$.

The second stage of the process consisted of reading the remaining 1,085 abstracts, based on which the other inclusion criteria (study design and target outcomes) were verified. After excluding review articles, articles on studies with an experimental design, and those that did not aim to study any of the selected mother-child outcomes, 39 studies were identified as adequate for inclusion in the present study. It was not possible to obtain access to two of these articles, so the final total was 37 studies. Figure 1, based on the proposal of the International Committee of Medical Journal Editors 11, presents a flowchart with a brief demonstration of all the stages in the article selection process for the present review.

To conduct an appraisal of the studies' methodological quality, each of the selected articles received a score, according to the criteria proposed by Downs \& Black ${ }^{12}$. The original checklist was adapted, ruling out the criteria related exclusively to intervention studies. Nineteen items were thus evaluated, allowing a maximum score of 20 points. Table 1 shows the items actually evaluated.

The studies were evaluated independently by two of the authors of the present review (E. B. S. and M. M. S.). The concordance in the scores assigned by the evaluators was also assessed using the Intra-Class Correlation Coefficient (ICC). The result was classified according to the scale proposed by Shrout 13 to measure concordance between different evaluation methods. This scale consists of five categories: virtually no concordance $(<0.1)$; weak (0.11-0.40); reasonable (0.41-0.60); moderate (0.61-0.80); and substantial (0.81-1.0). To rule on possible discordances in assigning scores to the articles, a third author (G. K.) was consulted.

The studies were organized according to the following: year of publication; country of origin; language; study design (cohort, case-control, cross-sectional); physical activity measurement instrument; sample size; maternal-child health outcomes; age of individuals studied (years); results; estimators used in the analysis and scoring of the methodological quality. 
Finally, the studies were grouped that found similar results as to a given outcome, with the objective of systematizing and facilitating the understanding of all the reviewed articles' findings. Next, the mean of the scores assigned to the studies included in each group was calculated. No articles were rejected because of methodological limitations, but the findings were considered more consistent in the studies that received higher scores.

\section{Results}

As shown in Table 2, the largest concentration of published articles (20) was in the 1990s. However, there was also an upward trend in publications on the subject, given that 11 studies were published in the first five years of the current decade. The majority of the studies were from the United States, and the only one conducted in Brazil 14 was also the only one not published in English.

Among the observational studies that evaluated possible associations between physical activity and maternal-child health outcomes, those with a prospective cohort design were the most common. Questionnaires were the most widely used instruments, reinforcing this as the most feasible option for measuring physical activity in epidemiological studies 15 . The sample sizes varied from 67 to 21,342 .

Table 3 shows that of the 37 articles reviewed, 15 used birth weight as the dependent variable, making this the most widely studied outcome. Among the articles that informed the participants' age (26), only five reported having included pregnant women younger than 18 years in their sample.

The mean methodological quality score was 15.8 points, with 9 and 20 points as the minimum and maximum obtained, respectively. The result of the comparison between the scores assigned to the studies by the different evaluators (ICC = 0.898; 95\%CI: 0.835-0.961) indicates a high level of concordance, having been classified as the highest (substantial concordance) in the qualification scale used.

Table 4 provides a synthesis of the principal results of the current review. Physical activity does in fact appear to reduce the risk of preeclampsia and gestational diabetes. The results were conflicting for other outcomes, like miscarriage and gestational weight gain control. The same was true for the fetal and childhood outcomes. Some studies do not describe a harmful association between physical activity in pregnancy and inadequate weight gain or prematurity, however some specific activities, like climbing stairs or working
Figure 1

Flowchart for article selection.

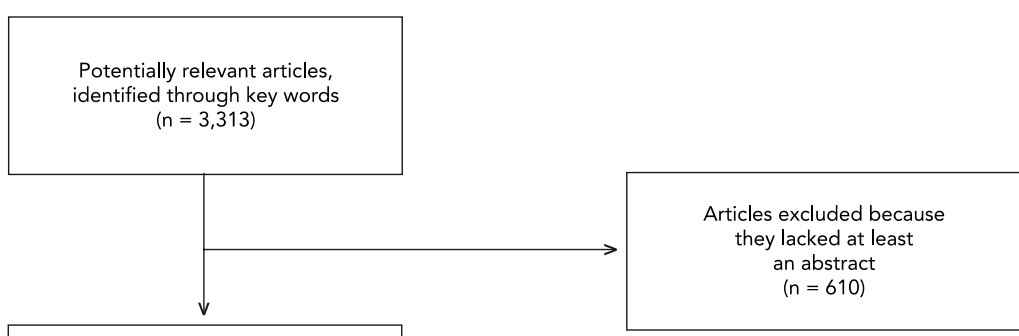

Potentially relevant articles, with at least an available abstract $(n=2,703)$
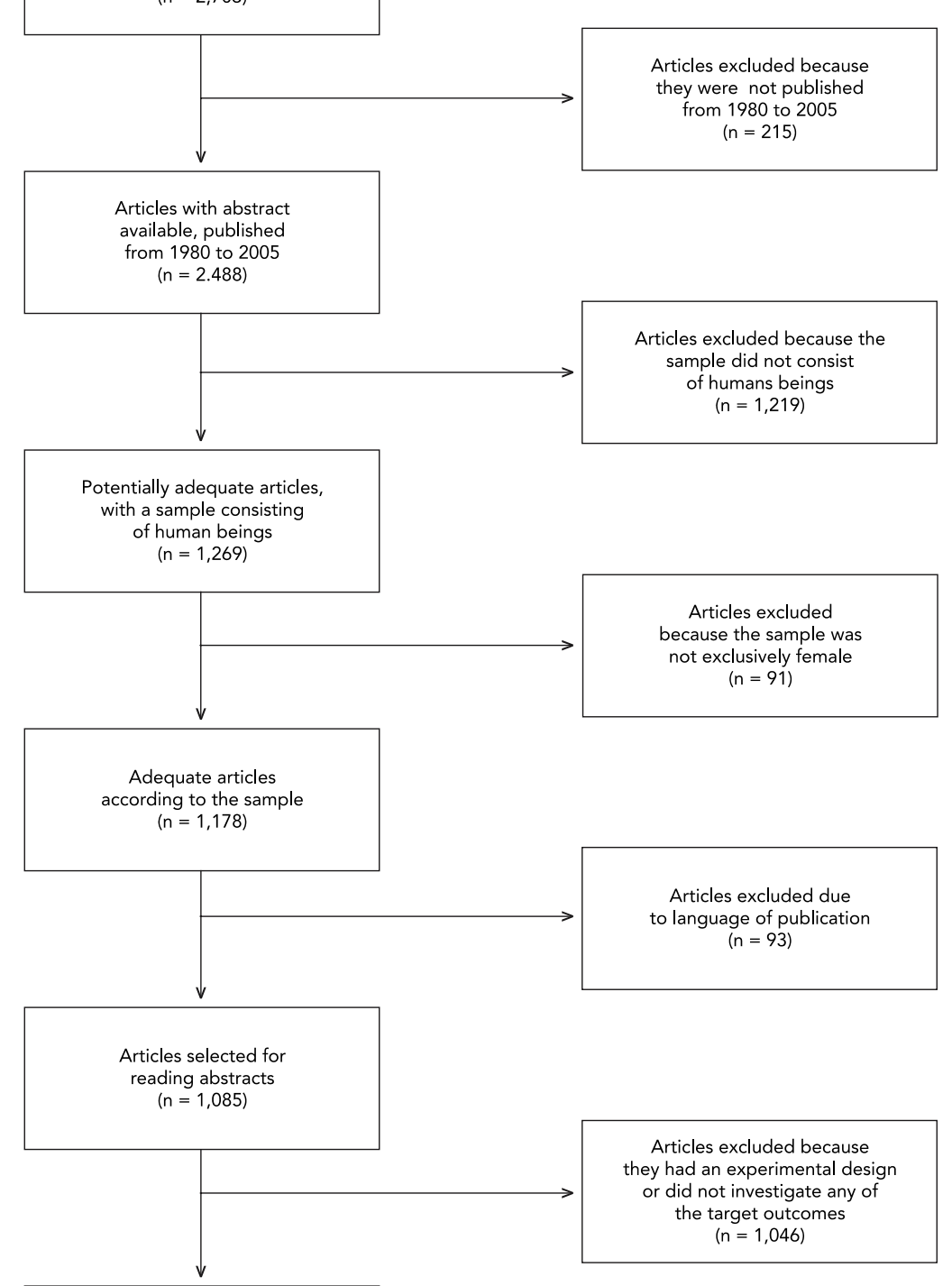

Articles selected for review $(n=39)$ 
Table 1

Downs \& Black criteria 12 used in the methodological evaluation of the articles.

\section{Criteria}

Clarity in the description of the study hypothesis or objective(s)

2. Definition of the target outcomes in the introduction or methods section

3. Description of the characteristics of the individuals included in the study

4. Description of principal confounders

5. Clarity in the description of the study's principal findings

6. Information on random data variability for the principal results

7. Description of characteristics of individuals lost to follow-up

8. Information on the real probability values for the principal results

9. Representativeness of planned sample

10. Representativeness of sample of individuals included in study

11. Clarity in the description of results not based on a priori hypotheses

12. Adjustment of analyses for different lengths of follow-up

13. Adequacy of statistical tests used to evaluate the principal results

14. Accuracy of the instruments used to measure the principal outcomes

15. Comparability among individuals from different groups

16. Equal recruitment periods for individuals from different groups

17. Inclusion of adjustment for principal confounders in the analysis

18. Consideration of losses to follow-up

19. Study power sufficient to detect an important effect, with a $5 \%$ significance level

All items scored 0 or 1 , except for description of principal confounders, scored 0,1 , or 2

for extended time on one's feet were identified as risk factors for these outcomes.

Few studies were identified on the effects of physical activity during pregnancy on fetal growth or development, length at birth, and mode of delivery.

\section{Discussion}

The production of scientific material on physical activity in pregnancy and its effects on maternalchild health outcomes has increased substantially, mainly in the last decade. According to some authors $3,8,16,17$, light-to-moderate exercise also provides health benefits during pregnancy, so women should be encouraged to maintain an active lifestyle during gestation. The results of some of the reviewed articles corroborate the opinion of these authors.

Considering both light and moderate leisure-time and occupational physical activities, physically active pregnant women show a decreased risk of developing preeclampsia $18,19,20$, hypertension 18,20 , and gestational diabetes mellitus $21,22,23$. However, in relation to the other maternal outcomes, the results tend to be conflicting. Four studies analyzed leisuretime physical activity in relation to miscarriage
24,25,26,27, and their results did not indicate an increased risk of this outcome among physically active pregnant women. Importantly, however, the studies that pointed to high-intensity occupational activity as a risk factor for miscarriage 28,29 were precisely those that showed greater methodological care, among other reasons because they based their conclusions on stratified analyses rather than crude data.

In a recent study 30 , physical activity was identified as one of three behavioral determinants for gestational weight gain control. However, the studies reviewed here did not agree as to the hypothesis of greater control of weight gain among pregnant women who exercise regularly. Five of the articles reviewed 27,31,32,33,34 studied this relationship, and three of them $27,32,33$ did not support the hypothesis. However, the three were precisely the ones with the lowest methodological quality scores or which failed to report the method used to evaluate this outcome.

Only four articles evaluated the effect of physical activity on mode of delivery, of which three $27,32,33$ failed to show significant differences between caesarian rates in women with and without exercise during pregnancy. Only one study 39 showed an association between physical activity and mode of delivery, suggesting a considerably increased risk of caesarian among sedentary pregnant women.

As for fetal and childhood outcomes, the results also failed to show a consensus, but once again light or moderate physical exercise was not identified as a risk factor. As for birth weight, numerous studies 25,32,33,36,37,38,39,40,41,42 showed that there is no increased risk of low birth weight for pregnant women who practice leisure-time physical activities.

Among the studies that analyzed physical activity as a predictive factor for low birth weight, some 27,34,43 showed a subtle association, but the mean weight of the newborns was not inadequate in any of the groups studied. However, other studies 42,44 identified some specific activities (e.g., doing laundry on standing on one's feet for hours on end), but not regular physical activity in general, as risk factors for inadequate birth weight.

The same was observed in studies in which the principal outcome was gestational age at delivery. Numerous articles either found no association or reported a protective effect for physical activity during pregnancy in relation to premature birth 27,32,33,34,36,40,42,45,46,47. Again, some specific activities, like climbing stairs and occupational activities that require standing for prolonged periods or cause fatigue and require 
Year, country of origin, language, design, instrument for measuring physical activity, and sample size of selected studies on physical activity in pregnancy and maternal-child health outcomes, 1980-2005.

\begin{tabular}{|c|c|c|c|c|c|c|}
\hline Author & Year & Country & Language & Design & Instrument & Sample \\
\hline Jarrett \& Sppelacy 36 & 1983 & USA & English & Cohort & Questionnaire & 67 \\
\hline Berkowitz et al. 45 & 1983 & USA & English & Case-control & Questionnaire & $175 * / 313 * \star$ \\
\hline Marcoux et al. 18 & 1989 & Canada & English & Case-control & Questionnaire & $172 * / 254 * / 505 * \star$ \\
\hline Clapp et al. 24 & 1989 & USA & English & Cohort & PEC & $49 * / 41 * / 29 * *$ \\
\hline Rabkin et al. 37 & 1990 & UK & English & Cohort & Questionnaire & 1,507 \\
\hline Klebanoff et al. 25 & 1990 & USA & English & Cohort & Questionnaire & 7,101 \\
\hline Rose et al. 38 & 1991 & USA & English & Cohort & Questionnaire & 21.342 \\
\hline Hatch et al. 39 & 1993 & USA & English & Cohort & Questionnaire & 462 \\
\hline Florack et al. 28 & 1993 & Netherlands & English & Cohort & Questionnaire & 170 \\
\hline Bell et al. 43 & 1995 & Australia & English & Cohort & Questionnaire & $58 * / 41 * \star$ \\
\hline Clapp \& Little 31 & 1995 & USA & English & Cohort & $\mathrm{CM}$ & 79 \\
\hline Henriksen et al. 48 & 1995 & Denmark & English & Cohort & Questionnaire & 4,259 \\
\hline Koemeester et al. 49 & 1995 & Netherlands & English & Cohort & Questionnaire & 116 \\
\hline Sternfeld et al. 32 & 1995 & USA & English & Cohort & Questionnaire & 388 \\
\hline Florack et al. 40 & 1995 & Netherlands & English & Cohort & Questionnaire & 128 \\
\hline Henriksen et al. 44 & 1995 & Denmark & English & Cohort & Questionnaire & 4,249 \\
\hline Spinillo et al. 58 & 1995 & Italy & English & Case-control & Questionnaire & $160 * / 320 * *$ \\
\hline Horns et al. 33 & 1996 & USA & English & Cohort & Questionnaire & $48 * / 53 * \star$ \\
\hline Schramm et al. 41 & 1996 & USA & English & Case-control & Questionnaire & $450 * / 782 * \star / 802 * / 794 * \star$ \\
\hline Spinillo et al. 50 & 1996 & Italy & English & Case-control & Questionnaire & $349 * / 698 * *$ \\
\hline Dye et al. 21 & 1997 & USA & English & Cross-sectional & Questionnaire & 12,799 \\
\hline Misra et al. 46 & 1998 & USA & English & Cohort & Questionnaire & 1,172 \\
\hline Hatch et al. 47 & 1998 & USA & English & Cohort & Questionnaire & $325 * / 232 * \star$ \\
\hline Alderman et al. 52 & 1998 & USA & English & Case-control & Questionnaire & $22 * / 37 * / 232 * \star$ \\
\hline Latka et al. 26 & 1999 & USA & English & Case-control & Questionnaire & $173 * / 173$ ** \\
\hline Bungun et al. 35 & 2000 & USA & English & Cohort & Questionnaire & 137 \\
\hline Campbell \& Mottola 59 & 2001 & Canada & English & Case-control & Questionnaire & $164 * / 365 * \star$ \\
\hline El Metwalli et al. 29 & 2001 & Egypt & English & Case-control & Questionnaire & $562 * / 1,200 * *$ \\
\hline Magann et al. 27 & 2002 & USA & English & Cohort & Questionnaire & 750 \\
\hline Carmichael et al. 51 & 2002 & USA & English & Case-control & Questionnaire & $414 * / 417 * *$ \\
\hline Leiferman \& Evenson 42 & 2003 & USA & English & Cross-sectional & Questionnaire & 9,089 \\
\hline Rao et al. 34 & 2003 & India & English & Cohort & Questionnaire & 797 \\
\hline Sorensen et al. 19 & 2003 & USA & English & Case-control & Questionnaire & $201 * / 383 \star \star$ \\
\hline Dempsey et al. 22 & 2004 & USA & English & Cohort & Questionnaire & 909 \\
\hline Saftlas et al. 20 & 2004 & USA & English & Cohort & Questionnaire & $44 * / 172 * / 2,422 * \star$ \\
\hline Dempsey et al. 23 & 2004 & USA & English & Case-control & Questionnaire & $155^{* / 386 * *}$ \\
\hline Takito et al. 14 & 2005 & Brazil & Portuguese & Cohort & Questionnaire & 152 \\
\hline
\end{tabular}

PEC: portable electrocardiography; CM: cardiac monitor

* Group of cases;

** Group of controls.

agility, were identified as risk factors for premature birth 25,48,49.

Little is known about the effects of physical activity on fetal development. Only two studies with this focus were identified, one with the objective of evaluating the effects of occupational activities on intrauterine growth retardation 50 and the other on leisure-time physical activities and the occurrence of neural tube defects 51 . Apparently, light occupational activities do not cause problems with the fetal growth rate, but the same was not reported for women who maintained moderate or high-intensity activities. According to the authors of the second 
Table 3

Principal outcomes, participants' age, principal results, and estimators of methodological score for selected studies on physical activity in pregnancy and maternal-child health outcomes, 1980-2005.

\begin{tabular}{|c|c|c|c|c|c|}
\hline Author & Outcomes & Age (years) * & Results & Estimators & Score ** \\
\hline $\begin{array}{l}\text { Jarrett \& } \\
\text { Spellacy } 36\end{array}$ & $\begin{array}{l}\text { Birth weight; } \\
\text { prematurity }\end{array}$ & $\begin{array}{c}24-36 \\
30 \pm 0.4\end{array}$ & $\begin{array}{l}\text { No statistically significant correlation between leisure-time physical activity } \\
\text { (total } k m \text { run) during either pregnancy as a whole, or in the third trimester } \\
\text { only, and birth weight ( } r=0.089 \text { and } r=0.145 \text {, respectively; } p \text { not significant } \\
\text { for either). Leisure-time physical activity during pregnancy also failed to } \\
\text { show a statistically significant correlation with gestational age at delivery } \\
\text { ( } r=0.060 \text {; } p \text { not significant). No report on method used to estimate } \\
\text { gestational age. Sample included premature newborns. }\end{array}$ & $\begin{array}{c}\text { Mean } \\
\text { correlation }\end{array}$ & 11 \\
\hline $\begin{array}{l}\text { Berkowitz } \\
\text { et al. } 45\end{array}$ & Prematurity & NR & $\begin{array}{l}\text { Women who maintained their occupational physical activities during } \\
\text { pregnancy showed lower risk }(\mathrm{OR}=0.68 ; 95 \% \mathrm{Cl} \text { : } 0.46-1.01) \text { of having } \\
\text { premature newborns. The same was true for women who practiced } \\
\text { leisure-time physical activity during the year prior to pregnancy }(\mathrm{OR}=0.67 \text {; } \\
95 \% \mathrm{Cl}: 0.46-0.97) \text { and during pregnancy }(\mathrm{OR}=0.53 ; 95 \% \mathrm{Cl} \text { : } 0.36-0.78) \text {, } \\
\text { as compared to women who did not practice any leisure-time physical } \\
\text { activity. No report on method used to estimate gestational age }\end{array}$ & OR & 16 \\
\hline $\begin{array}{l}\text { Marcoux } \\
\text { et al. } 18\end{array}$ & $\begin{array}{l}\text { Preeclampsia; } \\
\text { gestational } \\
\text { hypertension }\end{array}$ & $\begin{array}{c}26 \pm 4.8 \\
26.2 \pm 4.3 \\
26.2 \pm 4.2\end{array}$ & $\begin{array}{l}\text { Women with leisure-time physical activity during the first } 20 \text { weeks of pregnancy } \\
\text { showed lower risk of preeclampsia ( } \mathrm{RR}=0.67 ; 95 \% \mathrm{Cl} \text { : } 0.46-0.96 \text { ) and gestational } \\
\quad \text { hypertension ( } \mathrm{RR}=0.75 ; 95 \% \mathrm{Cl}: 0.54-1.05) \text {. The risk decreased as the } \\
\text { intensity of the leisure-time physical activities increased, both for preeclampsia } \\
(\mathrm{RR}=1.00 \text { [light]; } 0.77 \text { [moderate]; } 0.57 \text { [vigorous]; } \mathrm{p}=0.01 \text { ) and gestational } \\
\text { hypertension ( } \mathrm{RR}=1.00 \text { [light]; } 0.81 \text { [moderate]; and } 0.71 \text { [vigorous]; } \mathrm{p}=0.08 \text { ) }\end{array}$ & $\mathrm{RR}$ & 18 \\
\hline $\begin{array}{l}\text { Clapp } \\
\text { et al. } 24\end{array}$ & Miscarriage & $\begin{array}{l}25-38 \\
31 \pm 3\end{array}$ & $\begin{array}{l}\text { Miscarriage rates were } 17 \%, 18 \% \text {, and } 25 \% \text { for pregnant women who } \\
\text { practiced jogging, dance, and no leisure-time physical activity, respectively, } \\
\text { but the differences were not statistically significant. }\end{array}$ & Incidence & 9 \\
\hline $\begin{array}{l}\text { Rabkin } \\
\text { et al. } 37\end{array}$ & Birth weight & $\geq 15$ & $\begin{array}{l}\text { No evidence that occupational or domestic physical activities in pregnant } \\
\text { women were associated with low birth weight adjusted for gestational age. } \\
\text { After adjusting for confounders, occupational physical activity } \\
\text { (full-time employment) was associated with increased birth weight } \\
(12 \mathrm{~g} ; 95 \% \mathrm{Cl}:-39 \text { to } 63 \mathrm{~g}) \text {. Sample did not include premature newborns. }\end{array}$ & $\begin{array}{c}\beta \text { (linear } \\
\text { regression) }\end{array}$ & 20 \\
\hline $\begin{array}{l}\text { Klebanoff } \\
\text { et al. } 25\end{array}$ & $\begin{array}{l}\text { Birth weight; } \\
\text { prematurity }\end{array}$ & $\geq 16$ & $\begin{array}{l}\text { Physical activity (occupational, domestic, and leisure-time) was not } \\
\text { associated with birth weight after adjusting for confounders. Working on } \\
\text { one's feet for } 8 \text { hours or longer showed a slight increase in the risk of } \\
\text { prematurity (OR }=1.31 ; 95 \% \mathrm{Cl}: 1.01-1.71) \text {. Gestational age was estimated } \\
\text { based on date of last menstrual period and confirmed by some clinical } \\
\text { parameter (ultrasound or fetal heart sounds). Did not report whether the } \\
\text { sample included premature newborns. }\end{array}$ & OR & 14 \\
\hline $\begin{array}{l}\text { Rose } \\
\text { et al. } 38\end{array}$ & $\begin{array}{l}\text { Birth weight; } \\
\text { miscarriage }\end{array}$ & 25.6 & $\begin{array}{l}\text { Low birth weight rates were } 4.9 \%, 4.3 \% \text {, and } 4.3 \% \text { for pregnant women } \\
\text { classified according to intensity of physical activities as light, moderate, or } \\
\text { vigorous, respectively, but these differences were not statistically significant. } \\
\text { The same was observed for fetal death rates (1.3\%; } 1.0 \% \text {, and } 1.3 \% \text {, respectively). } \\
\text { Did not report whether the sample included premature newborns. }\end{array}$ & $\begin{array}{l}\text { Mean } \\
\text { incidence }\end{array}$ & 11 \\
\hline $\begin{array}{l}\text { Hatch } \\
\text { et al. } 39\end{array}$ & Birth weight & $\begin{array}{c}\geq 18 \\
27.1 \pm 4.3 \\
27.9 \pm 4.6\end{array}$ & $\begin{array}{l}\text { Pregnant women with light or moderate leisure-time physical activity } \\
\text { showed an increase of some } 100 \mathrm{~g} \text { in birth weight }(117 \mathrm{~g} ; 95 \% \mathrm{Cl}: 17-217 \mathrm{~g}) \text {, } \\
\text { compared to sedentary pregnant women. Pregnant women who reported } \\
\text { more vigorous exercise (energy expenditure approximately } 2,000 \mathrm{kcal} / \text { week) } \\
\text { showed an increase of some } 300 \mathrm{~g} \text { in birth weight ( } 276 \mathrm{~g} ; 95 \% \mathrm{Cl}: 54-497 \mathrm{~g}) \text {. } \\
\text { Did not report whether the sample included premature newborns. }\end{array}$ & $\begin{array}{c}\beta \text { (linear } \\
\text { regression) }\end{array}$ & 17 \\
\hline
\end{tabular}

(continues) 
Table 3 (continued)

\begin{tabular}{|c|c|c|c|c|c|}
\hline Author & Outcomes & Age (years) * & Results & Estimators & Score ** \\
\hline $\begin{array}{l}\text { Florack } \\
\text { et al. } 28\end{array}$ & Miscarriage & $18-39$ & $\begin{array}{l}\text { The duration (in hours) and speed demanded by occupational physical } \\
\text { activities were not associated with miscarriage. However, occupational physical } \\
\text { activities involving high biomechanical loads were associated with increased } \\
\text { miscarriage rate (OR }=3.1 ; 95 \% \mathrm{Cl}: 1.1-8.9)\end{array}$ & OR & 16 \\
\hline Bell et al. 43 & $\begin{array}{c}\text { Birth } \\
\text { weight }\end{array}$ & $\begin{array}{l}31.8 \pm 2.7 \\
31.6 \pm 4.7\end{array}$ & $\begin{array}{l}\text { Mean birth weight was lower among newborns in women who practiced } \\
\text { leisure-time physical activities more than } 4 \text { times a week }(3.049 \mathrm{~kg} \text { vs. } \\
3.364 \mathrm{~kg} ; \mathrm{p}<0.02) \text {, as compared to the control group. However, mean } \\
\text { birth weight was higher among newborns of women who exercised up to } \\
3 \text { times a week ( } 3.682 \mathrm{~kg} \text { vs. } 3.36 \mathrm{~kg} ; \mathrm{p}<0.01) \text { as compared to the control group. } \\
\text { Incidence of low birth weight was higher in women who practiced leisure-time } \\
\text { physical activities more than } 4 \text { times a week than in those who exercised up to } \\
3 \text { times }(22 \% \text { vs. } 3 \% ; p=0.03) \text {. Sample included premature newborns. }\end{array}$ & $\begin{array}{c}\text { Mean } \\
\text { incidence }\end{array}$ & 12 \\
\hline $\begin{array}{l}\text { Clapp \& } \\
\text { Little } 31\end{array}$ & $\begin{array}{l}\text { Gestational } \\
\text { weight gain }\end{array}$ & $31 \pm 0.3$ & $\begin{array}{l}\text { The difference between mean weight gain in the group of pregnant women } \\
\text { who practiced leisure-time physical activity }(13 \mathrm{~kg} \pm 0.5) \text { as compared to the } \\
\text { control group ( } 16 \pm 0.7) \text { was statistically significant. The correlations between } \\
\text { the amount of exercise and gestational weight gain were weak and } \\
\text { non-significant ( } r<0.15 \text { for the } 15-23,23-30 \text {, and } 30-37 \text {-week intervals). } \\
\text { Weight gain was monitored since before conception. }\end{array}$ & $\begin{array}{c}\text { Mean } \\
\text { correlation }\end{array}$ & 15 \\
\hline $\begin{array}{l}\text { Henriksen } \\
\text { et al. } 48\end{array}$ & Prematurity & NR & $\begin{array}{l}\text { Pregnant women who did not report occupational physical activities that } \\
\text { required standing or walking more than } 4 \text { hours showed higher odds of preterm } \\
\text { delivery (OR }=3.3 ; 95 \% \mathrm{Cl} \text { : } 1.4-8.0 \text { ) as compared to those who reported } 2 \text { hours } \\
\text { or less. Gestational age was estimated based on ultrasound (when available), } \\
\text { date of last menstrual period, or as recorded on the birth certificate. }\end{array}$ & OR & 18 \\
\hline $\begin{array}{l}\text { Koemeester } \\
\text { et al. } 49\end{array}$ & Prematurity & $21-46$ & $\begin{array}{c}\text { Daily duration (number of hours) of high-intensity occupational physical } \\
\text { activity showed a negative and statistically significant association with } \\
\text { age at birth }(\beta=-0.49 ; p<0.004) \text {. However, mean gestational length gestational } \\
\text { (in days) was not equal to prematurity for any of the groups studied. Did not } \\
\text { report the method used to estimate gestational age }\end{array}$ & $\begin{array}{l}\text { Mean } \beta \\
\text { (linear } \\
\text { regression) }\end{array}$ & 14 \\
\hline $\begin{array}{l}\text { Sternfeld } \\
\text { et al. } 32\end{array}$ & $\begin{array}{l}\text { Birth weight; } \\
\text { prematurity; } \\
\text { weight gain; } \\
\text { mode of delivery }\end{array}$ & $\begin{array}{c}18-42 \\
31.7 \pm 5.0\end{array}$ & $\begin{array}{l}\text { No statistically significant difference in mean birth weight for children } \\
\text { of women who practiced different levels of leisure-time physical activity, } \\
\text { before or during pregnancy (first, second, or third trimester) (largest difference } \\
\text { observed: } 121 \mathrm{~g} ; \mathrm{p}=0.29 \text { ). Likewise, mean length of pregnancy and mean } \\
\text { weight gain were similar between the different groups of pregnant women. } \\
\text { The caesarean rate also did not vary according to the level of maternal physical } \\
\text { activity. Gestational age was estimated based on the date of last menstrual period. } \\
\text { Gestational weight gain was calculated by subtracting the weight at the first prenatal } \\
\text { visit from weight at hospital admission for delivery, adjusted by the newborn's } \\
\text { weight. Did not report whether the sample included premature newborns. }\end{array}$ & Mean & 14 \\
\hline $\begin{array}{l}\text { Florack } \\
\text { et al. } 40\end{array}$ & $\begin{array}{l}\text { Prematurity; } \\
\text { birth weight }\end{array}$ & $18-39$ & $\begin{array}{l}\text { High-intensity occupational physical activities and those causing fatigue showed } \\
\text { the greatest effect on duration of pregnancy (minus } 18 \text { days), when the pace of } \\
\text { activities was high. None of the aspects of the occupational activities showed a } \\
\text { relevant influence on birth weight. Did not report the method used to estimate } \\
\text { gestational age. Did not report whether the sample included premature newborns. }\end{array}$ & $\begin{array}{c}\beta \text { (linear } \\
\text { regression) }\end{array}$ & 15 \\
\hline $\begin{array}{l}\text { Henriksen } \\
\text { et al. } 44\end{array}$ & Birth weight & NR & $\begin{array}{l}\text { Reduction }(-119 \mathrm{~g} ; 95 \% \mathrm{Cl}:-230 \text { to }-8 \mathrm{~g}) \text { in mean birth weight of newborns of } \\
\text { pregnant women who reported occupational physical activities that required } \\
\text { standing or walking for more than } 5 \text { hours as compared to pregnant women } \\
\text { reporting } 2 \text { hours or less. Sample included premature newborns. }\end{array}$ & $\begin{array}{c}\beta \text { (linear } \\
\text { regression) }\end{array}$ & 19 \\
\hline $\begin{array}{l}\text { Spinillo } \\
\text { et al. } 58\end{array}$ & Preeclampsia & $\begin{array}{l}28.4 \pm 5.4 \\
27.7 \pm 4.4\end{array}$ & $\begin{array}{c}\text { Pregnant women with moderate or vigorous occupational physical activities } \\
\text { were more susceptible to preeclampsia }(\mathrm{OR}=2.08 ; 95 \% \mathrm{Cl}: 1.11-3.88) \\
\text { than women performing light activities. }\end{array}$ & OR & 18 \\
\hline
\end{tabular}

(continues) 
Table 3 (continued)

\begin{tabular}{|c|c|c|c|c|c|}
\hline Author & Outcomes & Age (years) * & Results & Estimators & Score ** \\
\hline $\begin{array}{l}\text { Horns } \\
\text { et al. } 33\end{array}$ & $\begin{array}{l}\text { Weight gain; } \\
\text { birth weight; } \\
\text { prematurity; } \\
\text { mode of delivery }\end{array}$ & $\begin{array}{c}20-30 \\
27.2 \pm 3.8 \\
28.4 \pm 4.1\end{array}$ & $\begin{array}{l}\text { No statistically significant differences in mean weight gain }(16.3 \pm 5.26 \mathrm{~kg} \text { vs. } \\
17.3 \pm 5.85 \mathrm{~kg} ; \mathrm{p}>0.05) \text {, birth weight }(2.496 \pm 486 \mathrm{~g} \text { vs. } 3.467 \pm 434 \mathrm{~g} ; \mathrm{p}>0.05) \text {, } \\
\text { gestational age at delivery }(39.9 \pm 1.4 \text { vs. } 39.2 \pm 4.3 \text { weeks; } p>0.05) \text {, or caesarean } \\
\text { rate }(25 \% \text { vs. } 32 \% . p>0.05) \text { between groups of pregnant women with } \\
\text { leisure-time physical activity and sedentary pregnant women. Did not report } \\
\text { methods used to estimate gestational age or pre-gestational weight. } \\
\text { Sample included premature newborns. }\end{array}$ & $\begin{array}{c}\text { Mean } \\
\text { chi-squared }\end{array}$ & 11 \\
\hline $\begin{array}{l}\text { Schramm } \\
\text { et al. } 41\end{array}$ & Birth weight & NR & $\begin{array}{c}\text { The association between leisure-time physical activity and very low birth } \\
\text { weight showed a downward trend during the first }(\mathrm{OR}=0.70 ; 95 \% \mathrm{Cl}: 0.53-0.92) \text {, } \\
\text { second }(\mathrm{OR}=0.54 ; 95 \% \mathrm{Cl}: 0.40-0.74) \text {, and third ( } \mathrm{OR}=0.33 ; 95 \% \mathrm{Cl}: 0.20-0.53) \\
\text { trimesters. Sample included premature newborns. }\end{array}$ & OR & 17 \\
\hline $\begin{array}{l}\text { Spinillo } \\
\text { et al. } 50\end{array}$ & $\begin{array}{l}\text { Fetal growth } \\
\text { retardation }\end{array}$ & $\begin{array}{l}27.6 \pm 4.8 \\
27.6 \pm 4.3\end{array}$ & $\begin{array}{l}\text { After adjusting for confounders, the risk of intrauterine growth retardation } \\
\text { was similar for women who were unemployed versus employed at beginning of } \\
\text { pregnancy }(\mathrm{OR}=1.26 ; 95 \% \mathrm{Cl} \text { : } 0.86-1.83) \text {. However, risk of intrauterine growth } \\
\text { retardation was significantly higher in pregnant women who reported moderate } \\
\text { or vigorous occupational physical activity }(\mathrm{OR}=2.4 ; 95 \% \mathrm{Cl}: 1.36-4.21) \text {, } \\
\text { as compared to those reporting light activities. }\end{array}$ & OR & 18 \\
\hline Dye et al. 21 & $\begin{array}{l}\text { Gestational } \\
\text { diabetes } \\
\text { mellitus }\end{array}$ & NR & $\begin{array}{l}\text { After stratifying for pre-gestational BMI, leisure-time physical activity was } \\
\text { associated with a reduction in gestational diabetes mellitus only in pregnant } \\
\text { women with } \mathrm{BMI}>33 \mathrm{~kg} / \mathrm{m}^{2}(\mathrm{OR}=1.9 ; 95 \% \mathrm{Cl}: 1.2-3.1)\end{array}$ & OR & 16 \\
\hline $\begin{array}{l}\text { Misra } \\
\text { et al. } 46\end{array}$ & Prematurity & NR & 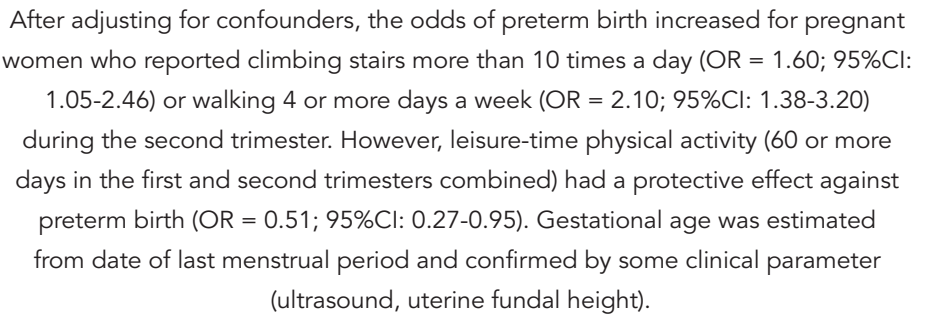 & OR & 16 \\
\hline $\begin{array}{l}\text { Hatch } \\
\text { et al. } 47\end{array}$ & Prematurity & $\begin{array}{c}\geq 18 \\
27.2 \pm 4.5 \\
27.7 \pm 4.5\end{array}$ & $\begin{array}{l}\text { No association observed between light or moderate leisure-time physical } \\
\text { activities and duration of pregnancy. Women who practiced vigorous leisure-time } \\
\text { physical activities showed a reduced risk ( } \mathrm{RR}=0.11 ; 95 \% \mathrm{Cl} \text { : } 0.02-0.81 \text { ) of preterm } \\
\text { delivery as compared to women who did not exercise. Gestational age was } \\
\text { estimated based on date of last menstrual period or ultrasound, when available. }\end{array}$ & $\mathrm{RR}$ & 17 \\
\hline $\begin{array}{l}\text { Alderman } \\
\text { et al. } 52\end{array}$ & Birth length & NR & $\begin{array}{l}\text { Moderate or vigorous leisure-time physical activity } 2 \text { hours a week or more in } \\
\text { any month of pregnancy was associated with decreased risk of large-for- } \\
\text { gestational-age newborns (OR }=0.3 ; 95 \% \mathrm{Cl} \text { : } 0.2-0.7) \text {, but was not associated } \\
\text { significantly with risk of small-for-gestational-age newborns } \\
\text { (OR }=0.8 ; 95 \% \mathrm{Cl}: 0.3-2.3) \text {. Sample included premature newborns. }\end{array}$ & OR & 14 \\
\hline Latka et al. 26 & Miscarriage & $\begin{array}{l}30,9 \pm 4.8 \\
32.2 \pm 5.8\end{array}$ & $\begin{array}{l}\text { Leisure-time physical activity during pregnancy was a protective factor against } \\
\qquad \text { miscarriage }(\mathrm{OR}=0.6 ; 95 \% \mathrm{Cl}: 0.3-0.9)\end{array}$ & OR & 15 \\
\hline $\begin{array}{l}\text { Bungun } \\
\text { et al. } 35\end{array}$ & $\begin{array}{l}\text { Mode of } \\
\text { delivery }\end{array}$ & $\begin{array}{c}17-40 \\
29.6 \pm 3.3 \\
28.4 \pm 4.6\end{array}$ & $\begin{array}{l}\text { After adjusting for confounders, there was an increased risk of caesarean } \\
\text { delivery for sedentary women (OR }=4.48 ; 95 \% \mathrm{Cl}: 1.2-16.2) \text { as compared } \\
\text { to those with leisure-time physical activity during pregnancy. }\end{array}$ & OR & 14 \\
\hline $\begin{array}{l}\text { Campbell \& } \\
\text { Mottola } 59\end{array}$ & $\begin{array}{l}\text { Birth } \\
\text { weight }\end{array}$ & NR & $\begin{array}{l}\text { Compared to pregnant women with leisure-time physical activity } 3 \text { to } 4 \text { times a } \\
\text { week in the third trimester, the odds of low birth weight were higher for those } \\
\text { who exercised twice or less }(\mathrm{OR}=2.64 ; 95 \% \mathrm{Cl}: 1.29-5.39) \text { and } 5 \text { times or } \\
\text { more }(\mathrm{OR}=4.61 ; 95 \% \mathrm{Cl}: 1.73-12.32) \text { per week. Did not report } \\
\text { whether the sample included premature newborns. }\end{array}$ & OR & 18 \\
\hline
\end{tabular}

(continues) 
Table 3 (continued)

\begin{tabular}{|c|c|c|c|c|c|}
\hline Author & Outcomes & Age (years) * & Results & Estimators & Score ** \\
\hline $\begin{array}{l}\text { El Metwalli } \\
\text { et al. } 29\end{array}$ & Miscarriage & $\begin{array}{l}28.2 \pm 7.3 \\
27.7 \pm 6.4\end{array}$ & $\begin{array}{c}\text { Intensity }(\mathrm{OR}=3.35 ; 95 \% \mathrm{Cl}: 2.71-4.12) \text {, pace }(\mathrm{OR}=2.24 ; 95 \% \mathrm{Cl}: 1.74-2.89) \text {, } \\
\text { duration in hours }(\mathrm{OR}=1.57 ; 95 \% \mathrm{Cl}: 1.25-1.97) \text {, and fatigue }(\mathrm{OR}=2.93 ; 95 \% \mathrm{Cl} \text { : } \\
2.26-3.81) \text { caused by occupational physical activities were identified as risk factors } \\
\text { for miscarriage in the sample of pregnant women. }\end{array}$ & OR & 18 \\
\hline $\begin{array}{l}\text { Magann } \\
\text { et al. } 27\end{array}$ & $\begin{array}{l}\text { Various } \\
\text { maternal-child } \\
\text { outcomes }\end{array}$ & $\begin{array}{l}23.7 \pm 4.9 \\
24.1 \pm 5 \\
25.4 \pm 4.7 \\
24.7 \pm 5.4\end{array}$ & $\begin{array}{l}\text { No significant difference in the proportion of cases of hypertension }(p=0.611) ; \\
\text { gestational diabetes; fetal death }(p=0.232) \text {; premature births }(p=0.414) ; \\
\text { caesareans }(p=0.621) \text {; mean weight gain }(p=0.391) \text {; or weeks of pregnancy } \\
(p=0.231) \text { between groups of pregnant women who exercised versus did } \\
\text { not exercise. On average, newborns of pregnant women who practiced vigorous } \\
\text { exercise were } 86.5 \mathrm{~g} \text { lighter }(p<0.001) \text { than those of pregnant women who did not } \\
\text { exercise. However, mean birth weight was not inadequate for any of the groups. } \\
\text { Did not report methods used to estimate gestational age or pre-gestational } \\
\text { weight. Sample included premature newborns. }\end{array}$ & $\begin{array}{c}\text { Mean } \\
\text { chi-squared }\end{array}$ & 18 \\
\hline $\begin{array}{l}\text { Carmichael } \\
\text { et al. } 51\end{array}$ & $\begin{array}{c}\text { Neural } \\
\text { tube defects }\end{array}$ & NR & $\begin{array}{l}\text { Newborns of pregnant women who practiced leisure-time physical activity } \\
\text { showed a } 30-50 \% \text { lower risk of neural tube defects as compared to sedentary } \\
\text { pregnant women. After adjusting for potential confounders, this association was } \\
\text { limited to pregnant women who did not use vitamin/mineral supplements during the } \\
\text { pre-gestational period; according to the physical activity score: } 1(\mathrm{OR}=0.94 ; 95 \% \mathrm{Cl} \text { : } \\
0.89-0.99), 5(\mathrm{OR}=0.72 ; 95 \% \mathrm{Cl}: 0.56-0.94) \text {, or } 10(\mathrm{OR}=0.52 ; 95 \% \mathrm{Cl}: 0.31-0.89) \text {. }\end{array}$ & OR & 18 \\
\hline $\begin{array}{l}\text { Leifeman \& } \\
\text { Evenson } 42\end{array}$ & $\begin{array}{l}\text { Birth weight; } \\
\text { prematurity }\end{array}$ & $15-49$ & $\begin{array}{l}\text { Women with no leisure-time physical activity before and during pregnancy were more } \\
\text { iusceptible to having newborns with very low birth weight }(\mathrm{OR}=1.75 ; 95 \% \mathrm{Cl}: 1.50-2.04) \\
\text { as compared to women who practiced leisure-time physical activity before and } \\
\text { during pregnancy. Previously active women who stopped their leisure-time physical } \\
\text { activities during pregnancy were more prone to low birth weight }(\mathrm{OR}=1.28 ; 95 \% \mathrm{Cl} \text { : } \\
\text { 1.05-1.56), or very low birth weight ( } \mathrm{OR}=2.05 ; 95 \% \mathrm{Cl}: 1.69-2.48) \text { as compared to } \\
\text { hose who remained active during pregnancy. No significant association between leisure- } \\
\text { ime physical activity and gestational age at time of delivery. Did not report method } \\
\text { used to estimate pre-gestational weight. Sample did not include premature newborns. }\end{array}$ & 4) & 17 \\
\hline Rao et al. 34 & $\begin{array}{l}\text { Various } \\
\text { maternal-child } \\
\text { outcomes }\end{array}$ & $\begin{array}{c}15-40 \\
20.8 \pm 2.9\end{array}$ & $\begin{array}{l}\text { Physical activity showed an inverse association with weight gain after the } 28 \text { th } \\
\text { week of pregnancy }(p=0.002) \text {. Vigorous physical activity before and during } \\
\text { pregnancy was associated with lower mean birth weight }(p=0.05 \text { and } 0.02 \text {, } \\
\text { respectively); newborn head circumference }(p=0.005 \text { and } 0.009 \text {, respectively), and } \\
\text { newborn arm circumference }(p=0.03 \text { and } 0.01 \text {, respectively). However, mean birth } \\
\text { weight was not inadequate for any of the groups. Physical activity did not influence } \\
\text { the incidence of prematurity or neonatal death. Gestational age was estimated from } \\
\text { the date of last menstrual period (or ultrasound, if there was more than a } 2 \text { week } \\
\text { discrepancy). Gestational weight gain was monitored since before conception. } \\
\text { Sample did not include premature newborns. }\end{array}$ & Mean & 15 \\
\hline $\begin{array}{l}\text { Sorensen } \\
\text { et al. } 19\end{array}$ & Preeclampsia & NR & $\begin{array}{l}\text { Women with leisure-time physical activity during the first } 20 \text { weeks of pregnancy } \\
\text { showed a } 35 \% \text { reduction in risk of preeclampsia (OR }=0.65 ; 95 \% \mathrm{Cl}: 0.43-0.99) \text { as } \\
\text { compared to those classified as inactive. Women with leisure-time physical activity } \\
\text { during the year prior to pregnancy showed a slightly lower reduction }(\mathrm{OR}=0.67 \text {; } \\
95 \% \mathrm{Cl} \text { : } 0.42-1.08) \text {. For those with leisure-time physical activity before and during } \\
\text { pregnancy, the reduction in risk of preeclampsia was significantly greater } \\
\qquad(\mathrm{OR}=0.59 ; 95 \% \mathrm{Cl}: 0.35-0.98)\end{array}$ & OR & 18 \\
\hline $\begin{array}{l}\text { Dempsey } \\
\text { et al. } 22\end{array}$ & $\begin{array}{c}\text { Gestational } \\
\text { diabetes mellitus }\end{array}$ & $\geq 18$ & $\begin{array}{l}\text { Compared to women classified as inactive, those with leisure-time physical activity } \\
\text { during the year prior to pregnancy showed a } 66 \% \text { reduction in risk of gestational } \\
\text { diabetes mellitus ( } R R=0.44 ; 95 \% \mathrm{Cl}: 0.21-0.91 \text { ). Women with leisure-time physical } \\
\text { activity during pregnancy showed a } 31 \% \text { reduction in risk of gestational diabetes } \\
\text { mellitus, but this association was not statistically significant }(\mathrm{RR}=0.69 ; 95 \% \mathrm{Cl} \text { : } \\
0.37-1.29) \text {. Women with leisure-time physical activity before and during } \\
\text { pregnancy showed a larger reduction in risk of gestational diabetes mellitus } \\
\text { ( } \mathrm{RR}=0.31 ; 95 \% \mathrm{Cl}: 0.12-0.79) \text {. }\end{array}$ & RR & 16 \\
\hline
\end{tabular}

(continues) 
Table 3 (continued)

\begin{tabular}{|c|c|c|c|c|c|}
\hline Author & Outcomes & Age (years) & Results & Estimators & Score ** \\
\hline $\begin{array}{l}\text { Saftlas } \\
\text { et al. } 20\end{array}$ & $\begin{array}{c}\text { Preeclampsia; } \\
\text { gestational } \\
\text { hypertension }\end{array}$ & $\begin{array}{c}31.4 \pm 4.4 \\
30.8 \pm 4 \\
30.5 \pm 4.7\end{array}$ & $\begin{array}{l}\text { Reduced risk of preeclampsia in pregnant women with leisure-time physical } \\
\text { activity }(\mathrm{OR}=0.66 ; 95 \% \mathrm{Cl}: 0.35-1.22) \text { or non-sedentary occupational physical } \\
\text { activities (OR }=0.71 ; 95 \% \mathrm{Cl}: 0.37-1.36) \text {. No association between leisure-time } \\
\text { or occupational physical activity and gestational hypertension. }\end{array}$ & OR & 17 \\
\hline $\begin{array}{l}\text { Dempsey } \\
\text { et al. } 23\end{array}$ & $\begin{array}{c}\text { Gestational } \\
\text { diabetes mellitus }\end{array}$ & 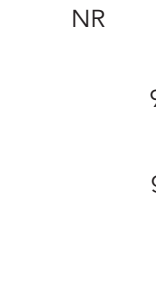 & $\begin{array}{l}\text { Women with any type of leisure-time physical activity in the year prior to pregnancy } \\
\text { showed a } 55 \% \text { reduction in risk of gestational diabetes mellitus (OR }=0.45 \text {; } \\
95 \% \mathrm{Cl} \text { : } 0.28-0.74) \text {, compared to sedentary women. Women with leisure-time physical } \\
\text { activity during the first } 20 \text { weeks of pregnancy showed a } 48 \% \text { reduction in risk of } \\
\text { gestational diabetes mellitus (OR }=0.52 ; 95 \% \mathrm{Cl} \text { : } 0.33-0.80) \text {, and women with leisure- } \\
\text { time physical activity before and during pregnancy showed the largest reduction } \\
\text { in risk of gestational diabetes mellitus (OR }=0.40 ; 95 \% \mathrm{Cl}: 0.23-0.68) \text {. }\end{array}$ & OR & 17 \\
\hline $\begin{array}{l}\text { Takito } \\
\text { et al. } 14\end{array}$ & $\begin{array}{l}\text { Birth } \\
\text { weight }\end{array}$ & $>18$ & $\begin{array}{l}\text { After adjusting for confounders, leisure-time physical activity (walking for at } \\
\text { least } 50 \text { minutes a day) in the first trimester of pregnancy was identified as a } \\
\text { protective factor against inadequate birth weight (OR }=0.44 ; 95 \% \mathrm{Cl}: 0.20-0.98) \text {. } \\
\text { Standing for more than } 2.5 \text { hours at a time during the second trimester showed } \\
\text { a sharp risk for inadequate birth weight ( } \mathrm{OR}=3.23 ; 95 \% \mathrm{Cl}: 1.30-7.99) \text {. Among } \\
\text { the occupational physical activities that require erect posture, only doing laundry } \\
3 \text { or more times a week in the second trimester was significantly associated with } \\
\text { inadequate birth weight (OR }=3.49 ; 95 \% \mathrm{Cl}: 1.59-7.64) \text {. Did not report whether the } \\
\text { sample included premature newborns. }\end{array}$ & OR & 16 \\
\hline
\end{tabular}

BMI: body mass index; NR: not reported; OR: odds ratio; RR: relative risk.

* Information on participants' age was not provided in a standardized way by the studies. Such information was available as age brackets, mean age (for the different target groups or the entire sample), and standard deviations, when possible;

** Methodological quality score of the reviewed studies, according to the Downs \& Black criteria 12.

study, leisure-time physical activity appears to play a protective role against the occurrence of neural tube malformations.

As for length at birth, only one study was located 52, showing a decreased risk of large-forgestational-age neonates among physically active pregnant women, but the study's methodological quality score was not one of the highest.

Two selected articles were not included in the present review due to lack of access to the full text. One of the articles 53 investigates the effects of physical activity during pregnancy on labor among mothers of premature infants. Based on the results, the authors suggest that physical activity in pregnancy can substantially reduce (by up to 32 minutes) the duration of the second stage of labor, as well as the risk of obstetric complications in this group of women. The second article 54 describes the results of two studies that investigated the effects of jogging during pregnancy. In the first, no significant differences were observed between the groups of mothers who jogged (versus those who did not jog) in relation to gestational weight gain and birth weight. Few obstetric complications were also observed in the group of women who jogged during pregnancy.
The second study refers to the effects of jogging on maternal and fetal heart rates, and was outside the scope of the current review.

Although more than a decade has transpired since the first recommendation by the American College of Obstetricians and Gynecologists (ACOG) 2 in favor of physical activity during pregnancy, little knowledge has been accumulated on the effects of this continuous practice on outcomes like fetal development, length at birth, miscarriage, mode of delivery, and gestational weight gain control. However, for other outcomes like birth weight and prematurity, a consensus appears to be taking shape that light or moderate leisure-time or occupational physical activities do not constitute a risk factor, and in some cases may be considered a protective factor.

Although displaying some important limitations, several studies received high scores for their methodological quality. The internal consistency of the scores assigned to the studies gave greater robustness to this evaluation. Among the Downs \& Black criteria 12 considered here, the principal limitations observed in the studies were: failing to commenting on the sample's representativeness, not presenting the list of 
Number of studies and their mean score \# according to type of association observed between physical activity in pregnancy and maternal-child health outcomes, 1980-2005

\begin{tabular}{|c|c|c|c|c|c|c|c|c|c|c|c|c|}
\hline \multirow[t]{3}{*}{ Outcomes } & \multicolumn{4}{|c|}{ Beneficial effect } & \multicolumn{4}{|c|}{ No effect } & \multicolumn{4}{|c|}{ Harmful effect } \\
\hline & \multicolumn{2}{|c|}{$\begin{array}{c}\text { Leisure-time } \\
\text { physical activity }\end{array}$} & \multicolumn{2}{|c|}{$\begin{array}{l}\text { Occupational } \\
\text { physical activity }\end{array}$} & \multicolumn{2}{|c|}{$\begin{array}{l}\text { Leisure-time } \\
\text { physical activity }\end{array}$} & \multicolumn{2}{|c|}{$\begin{array}{l}\text { Occupational } \\
\text { physical activity }\end{array}$} & \multicolumn{2}{|c|}{$\begin{array}{l}\text { Leisure-time } \\
\text { physical activity }\end{array}$} & \multicolumn{2}{|c|}{$\begin{array}{l}\text { Occupational } \\
\text { physical activity }\end{array}$} \\
\hline & $\mathbf{n}$ & $\begin{array}{l}\text { Mean } \\
\text { score }\end{array}$ & $\mathrm{n}$ & $\begin{array}{l}\text { Mean } \\
\text { score }\end{array}$ & $\mathrm{n}$ & $\begin{array}{l}\text { Mean } \\
\text { score }\end{array}$ & $\mathbf{n}$ & $\begin{array}{l}\text { Mean } \\
\text { score }\end{array}$ & $\mathbf{n}$ & $\begin{array}{l}\text { Mean } \\
\text { score }\end{array}$ & $\mathbf{N}$ & $\begin{array}{l}\text { Mean } \\
\text { score }\end{array}$ \\
\hline Preeclampsia & 3 & 17.7 & 1 & 17.0 & - & & - & - & - & - & 1 & 18.0 \\
\hline Gestational hypertension & 1 & 18.0 & - & - & 2 & 17.5 & 1 & 17.0 & - & - & - & - \\
\hline Gestational diabetes & 3 & 16.3 & - & - & 1 & 18.0 & - & - & - & - & - & - \\
\hline Miscarriage & 1 & 15.0 & - & - & 4 & 13.2 & 3 & 14.0 & - & - & 2 * & 17.0 \\
\hline Weight gain & 2 & 15.0 & 1 & 15.0 & 3 & 14.3 & - & - & - & - & - & - \\
\hline Mode of delivery & 1 & 14.0 & - & - & 3 & 14.3 & - & - & - & - & - & - \\
\hline Fetal growth \& development & 1 & 18.0 & - & - & - & - & - & - & - & - & $1 *$ & 18.0 \\
\hline Birth length & 1 & 14.0 & - & - & - & - & - & - & - & - & - & - \\
\hline Birth weight & 6 & 16.2 & 1 & 20.0 & 7 & 13.4 & 5 & 15.0 & $2 * \star$ & 15.0 & $2 * \star$ & 17.5 \\
\hline Prematurity & 3 & 16.3 & 1 & 16.0 & 7 & 14.7 & 2 & 14.5 & $1 *$ & 16.0 & 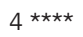 & 15.7 \\
\hline
\end{tabular}

\# Methodological quality score of reviewed studies, according to Downs \& Black criteria 12

* Refers to studies that identified moderate to vigorous leisure-time physical activity or certain characteristics of occupational physical activities, for example, standing for long hours, as a risk factor, but not physical activities as a whole. The number of asterisks represents the studies with this characteristic.

Note: the same study can appear in more than one box in the Table.

the principal confounders and/or not including them in the analysis or not making this clear in the text, and not presenting the study's power to detect differences between the groups. The lowest scores were assigned to articles from the first two decades, suggesting that current studies are possibly being developed and reported with greater methodological care. Since this review was conducted in databases with rigorous indexing criteria, incorporating only articles published in certain languages, a publication bias cannot be ruled out.

Importantly, the description was rather precarious for the individuals comprising the samples in some of the studies reviewed here. Gottlieb 55, in a brief communication, suggests that some of the contradictions observed in the results of studies on physical activity in pregnancy may result from the different methodologies, particularly differences among the individuals comprising the samples.

In relation to the sample group, Dye \& Oldenettel 56 already indicated that analyses based on special groups like elite athletes may lead to an error in their comparison with sedentary pregnant women. The present review did not include studies that specifically analyzed pregnant athletes. Even so, it is not difficult to imagine that physically fit pregnant women, with physical activity incorporated into their lives as a daily practice since before pregnancy, may constitute a particular group, quite different from sedentary pregnant women. The results of some of the studies reviewed here even demonstrated these peculiarities by presenting different effects for groups of pregnant women who practiced leisure-time physical activities before pregnancy and those who began exercising during gestation. Therefore, the knowledge should be interpreted in light of this consideration.

Another aspect that merits reflection is the fact that the Downs \& Black criteria 12 do not include any assessment of the quality of the research instruments or the exposure measurement process. Since most of the studies used questionnaires to assess the target phenomena, it would be interesting for future reviews to systematically incorporate these important methodological aspects.

The authors of the current review believe that the ACOG recommendations, especially those referring to care in the monitoring and follow-up of pregnant women, should be considered the baseline for any proposal to encourage a more active lifestyle for women experiencing the unique physiological moment of pregnancy.

In addition, the information presented here on what are considered risk activities for the oc- 
currence of certain maternal-child health outcomes should be widely publicized and especially incorporated into prenatal care guidelines. We were only able to find vague guidelines from the Brazilian Ministry of Health concerning physical activity during pregnancy. The Ministry's technical handbook for prenatal care and postpartum follow-up 57 suggests that physical activities should be encouraged to assist control of blood pressure and that pregnant women presenting gestational diabetes mellitus should exercise regularly. However, there was no recommendation for pregnant women as to exercise modality or even frequency.

Although light-to-moderate physical activity does not appear to be significantly associated with increased risk, more studies are needed to fill the gaps identified here. Most studies in the

\section{Resumo}

Realizou-se uma revisão sistemática da literatura com o objetivo de investigar o efeito da prática de atividade física durante a gestação em desfechos da saúde materno-infantil. A busca contemplou artigos publicados entre 1980 e 2005 nas bases de dados MEDLINE e LILACS utilizando-se as palavras-chave: physical activity; physical exercise; pregnancy e gestation. Foi feita uma avaliação da qualidade metodológica dos 37 artigos selecionados. Parece consenso que a prática de atividades físicas de intensidade leve ou moderada não consiste em fator de risco para alguns desfechos e pode representar fator de proteção. Contudo, alguns estudos encontraram associação entre atividades específicas, como subir escadas ou permanecer de pé por períodos prolongados e o peso inadequado do recém-nascido, prematuridade e aborto espontâneo. Poucos estudos encontraram associação entre a prática de atividades físicas e o ganho ponderal, tipo de parto e o desenvolvimento fetal. Novos estudos devem ser desenvolvidos com o objetivo de preencher essas lacunas, bem como propor recomendações acerca da intensidade, duração e freqüência das atividades físicas a serem realizadas durante a gestação.

Atividade Motora; Exercício Físico; Gravidez; Revisão current review lacked any kind of standardization as to the type of activities evaluated, merely defining them as either leisure-time or occupational physical activities. It thus becomes practically impossible to compare the studies' results, so that discussion of the findings becomes basically descriptive.

The definition of physical activity encompasses a series of aspects including all voluntary activities, like leisure-time, domestic, occupational, and commuting activities 60 . When counseling the pregnant woman as to physical activity during pregnancy, it is thus necessary to mention the frequency, intensity, and duration of such activities. These are the points that appear to lack the most information, since neither the ACOG nor the Brazilian Ministry of Health guidelines mention such specifications.

\section{Contributors}

M. M. Schlüssel participated in all stages of the study, from planning the review to the literature survey, article selection, Downs \& Black scoring, analysis of the results, and drafting of the article. E. B. Souza contributed to the literature review, Downs \& Black scoring, and revisions of all the versions of the manuscript. M. E. Reichenheim collaborated in the methodological review and final revision of the article. G. Kac participated in planning the review and orientation and revision of all versions of the article.

\section{Acknowledgments}

The authors wish to thank the Brazilian National Research Council (CNPq) and the Coordinating Division for the Support of Graduate Studies (CAPES). 


\section{References}

1. Batista DC, Chiara VL, Gugelmin SA, Martins PD Atividade física e gestação: saúde da gestante não atleta e crescimento fetal. Rev Bras Saúde Matern Infant 2003; 3:151-8.

2. American College of Obstetricians and Gynecologists. Exercise during pregnancy and the postpartum period. Washington DC: American College of Obstetricians and Gynecologists; 1994.

3. Lima FR, Oliveira N. Gravidez e exercício. Rev Bras Reumatol 2005; 45:188-90.

4. ACOG Committee on Obstetric Practice. Committee opinion \#267: exercise during pregnancy and the postpartum period. Obstet Gynecol 2002; 99:171-3.

5. Shills ME, Olson JA, Shike M, Ross AC, organizadores. Tratado de nutrição moderna na saúde e na doença. São Paulo: Edições Manole; 2003.

6. Hills AP, Byrne NM. Physical activity in the management of obesity. Clin Dermatol 2004; 22:315-8.

7. Gallup E. Aspectos legais da prescrição de exercícios para a gravidez. Seção III: aplicações práticas. In: Artal R, Wiswell AR, Drinkwater LR, organizadores. O exercício na gravidez. São Paulo: Edições Manole; 1999. p. 293-8.

8. Morris SN, Johnson NR. Exercise during pregnancy: a critical appraisal of the literature. J Reprod Med 2005; 50:181-8.

9. Downs DS, Hausenblas HA. Women's exercise beliefs and behaviors during their pregnancy and postpartum. J Midwifery Women's Health 2004; 49:138-44.

10. Clarke PE, Gross H. Women's behavior, beliefs and information sources about physical exercise in pregnancy. Midwifery 2004; 20:133-41.

11. International Committee of Medical Journal Editors. Improving the quality of reports of metaanalyses of randomised controlled trials: the QUOROM statement checklist. http://www. consort-statement.org/QUOROM.pdf (accessed on 14/May/2007).

12. Downs SH, Black N. The feasibility of creating a checklist for the assessment of the methodological quality both of randomised and non-randomised studies of health care interventions. J Epidemiol Community Health 1998; 52:377-84.

13. Shrout PE. Measurement reliability and agreement in psychiatry. Stat Methods Med Res 1998; 7:301-17.

14. Takito MY, Benício MHD, Latorre MRDO. Postura materna durante a gestação e sua influência sobre o peso ao nascer. Rev Saúde Pública 2005; 39: 325-32.

15. Reis RS, Petroski EL, Lopes AS. Medidas da atividade física: revisão de métodos. Rev Bras Cineantropom Desempenho Hum 2000; 2:89-96.

16. Dewey KG, McCrory MA. Effects of dieting and physical activity on pregnancy and lactation. Am J Clin Nutr 1994; 59(2 Suppl):446S-53S.

17. Clapp JF 3rd. Exercise during pregnancy: a clinical update. Clin Sports Med 2000; 19:273-83.

18. Marcoux S, Brisson J, Fabia J. The effect of leisure time physical activity on the risk of pre-eclampsia and gestational hypertension. J Epidemiol Community Health 1989; 43:147-52.
19. Sorensen TK, Williams MA, Lee IM, Dashow EE, Thompson ML, Luthy DA. Recreational physical activity during pregnancy and risk of preeclampsia. Hypertension 2003; 41:1273-80.

20. Saftlas AF, Logsden-Sackett N, Wang W, Woolson R, Bracken MB. Work, leisure-time physical activity, and risk of preeclampsia and gestational hypertension. Am J Epidemiol 2004; 160:758-65.

21. Dye TD, Knox KL, Artal R, Aubry RH, Wojtowycz MA. Physical activity, obesity, and diabetes in pregnancy. Am J Epidemiol 1997; 146:961-5.

22. Dempsey JC, Sorensen TK, Williams MA, Lee IM, Miller RS, Dashow EE, et al. Prospective study of gestational diabetes mellitus risk in relation to maternal recreational physical activity before and during pregnancy. Am J Epidemiol 2004; 159: 663-70.

23. Dempsey JC, Butler CL, Sorensen TK, Lee IM, Thompson ML, Miller RS, et al. A case-control study of maternal recreational physical activity and risk of gestational diabetes mellitus. Diabetes Res Clin Pract 2004; 66:203-15.

24. Clapp JF 3rd. The effects of maternal exercise on early pregnancy outcome. Am J Obstet Gynecol 1989; 161(6 Pt 1):1453-7.

25. Klebanoff MA, Shiono PH, Carey JC. The effect of physical activity during pregnancy on preterm delivery and birth weight. Am J Obstet Gynecol 1990; 163:1450-6.

26. Latka M, Kline J, Hatch M. Exercise and spontaneous abortion of known karyotype. Epidemiology 1999; 10:73-5.

27. Magann EF, Evans SF, Weitz B, Newnham J. Antepartum, intrapartum, and neonatal significance of exercise on healthy low-risk pregnant working women. Obstet Gynecol 2002; 99:466-72.

28. Florack EIM, Zielhuis GA, Pellegrino JEMC, Rolland R. Occupational physical activity and the occurrence of spontaneous abortion. Int J Epidemiol 1993; 22:878-84.

29. El Metwalli AGA, Badawy AM, El Baghdadi LA, El Wehady A. Occupational physical activity and pregnancy outcome. Eur J Obstet Gynecol Reprod Biol 2001; 100:41-5.

30. Olson CM, Strawderman MS. Modifiable behavioral factors in a biopsychosocial model predict inadequate and excessive gestational weight gain. J Am Dietetic Assoc 2003; 103:48-54.

31. Clapp JF 3rd, Little KD. Effect of recreational exercise on pregnancy weight gain and subcutaneous fat deposition. Med Sci Sports Exerc 1995; 27: 170-7.

32. Sternfeld B, Quesenberry Jr CP, Eskenazi B, Newman LA. Exercise during pregnancy and pregnancy outcome. Med Sci Sports Exerc 1995; 27:634-40.

33. Horns PN, Ratcliffe LP, Leggett JC, Swanson MS. Pregnancy outcomes among active and sedentary primiparous women. J Obstet Gynecol Neonatal Nurs 1996; 25:49-54.

34. Rao S, Kanade A, Margetts BM, Yajnik CS, Lubree $\mathrm{H}$, Rege S, et al. Maternal activity in relation to birth size in rural India. The Pune Maternal Nutrition Study. Eur J Clin Nutr 2003; 57:531-42. 
35. Bungum TJ, Peaslee DL, Jackson AW, Perez MA. Exercise during pregnancy and type of delivery in nulliparae. J Obstet Gynecol Neonatal Nurs 2000; 29:258-64.

36. Jarrett JC 2nd, Spellacy WN. Jogging during pregnancy: an improved outcome? Obstet Gynecol 1983; 61:705-9.

37. Rabkin CS, Anderson HR, Bland JM, Brooke OG, Chamberlain G, Peacock JL. Maternal activity and birth weight: a prospective, population-based study. Am J Epidemiol 1990; 131:522-31.

38. Rose NC, Haddow JE, Palomaki GE, Knight GJ. Self-rated physical activity level during the second trimester and pregnancy outcome. Obstet Gynecol 1991; 78:1078-80.

39. Hatch MC, Shu XO, McLean DE, Levin B, Begg M, Reuss L, et al. Maternal exercise during pregnancy, physical fitness, and fetal growth. Am J Epidemiol 1993; 137:1105-14.

40. Florack EI, Pellegrino AE, Zielhuis GA, Rolland R. Influence of occupational physical activity on pregnancy duration and birthweight. Scand J Work Environ Health 1995; 21:199-207.

41. Schramm WF, Stockbauer JW, Hoffman HJ. Exercise, employment, other daily activities, and adverse pregnancy outcomes. Am J Epidemiol 1996; 143:211-8.

42. Leiferman JA, Evenson KR. The effect of regular leisure physical activity on birth outcomes. Matern Child Health J 2003; 7:59-64.

43. Bell RJ, Palma SM, Lumley JM. The effect of vigorous exercise during pregnancy on birth-weight. Aust N Z J Obstet Gynaecol 1995; 35:46-51.

44. Henriksen TB, Hedegaard M, Secher NJ. Standing and walking at work and birthweight. Acta Obstet Gynecol Scand 1995; 74:509-16.

45. Berkowitz GS, Kelsey JL, Holford TR, Berkowitz RL. Physical activity and the risk of spontaneous preterm delivery. J Reprod Med 1983; 28:581-8.

46. Misra DP, Strobino DM, Stashinko EE, Nagey DA, Nanda J. Effects of physical activity on preterm birth. Am J Epidemiol 1998; 147:628-535.

47. Hatch M, Levin B, Shu XO, Susser M. Maternal leisure-time exercise and timely delivery. Am J Public Health 1998; 88:1528-33.
48. Henriksen TB, Hedegaard M, Secher NJ, Wilcox AJ. Standing at work and preterm delivery. Br J Obstet Gynaecol 1995; 102:198-206.

49. Koemeester AP, Broersen JPJ, Treffers PE. Physical work load and gestational age at delivery. Occup Environ Medicine 1995; 52:313-5.

50. Spinillo A, Capuzzo E, Baltaro F, Piazzi G, Nicola $\mathrm{S}$, Iasci A. The effect of work activity in pregnancy on the risk of fetal growth retardation. Acta Obstet Gynecol Scand 1996; 75:531-6.

51. Carmichael SL, Shaw GM, Neri E, Schaffer DM, Selvin S. Physical activity and risk of neural tube defects. Matern Child Health J 2002; 6:151-7.

52. Alderman BW, Zhao $\mathrm{H}$, Holt VL, Watts $\mathrm{DH}$, Beresford SAA. Maternal physical activity in pregnancy and infant size for gestational age. Ann Epidemiol 1998; 8:513-9.

53. Botkin C, Driscoll CE. Maternal aerobic exercise: newborn effects. Fam Pract Res J 1991; 11:387-93.

54. Dale E, Mullinax KM, Bryan DH. Exercise during pregnancy: effects on the fetus. Can J Appl Sport Sci 1982; 7:98-103.

55. Gottlieb S. Exercise during pregnancy may increase birth weight. BMJ 2001; 322:193.

56. Dye TD, Oldenettel D. Physical activity and risk of preterm labor: an epidemiological review and synthesis of recent literature. Semin Perinatol 1996; 20:334-9.

57. Área Técnica de Saúde da Mulher, Departamento de Ações Programáticas Estratégicas, Secretaria de Atenção à Saúde, Ministério da Saúde. Pré-natal e puerpério: atenção qualificada e humanizada - manual técnico. Brasília: Ministério da Saúde; 2005.

58. Spinillo A, Capuzzo E, Colonna L, Piazzi G, Nicola $S$, Baltaro F. The effect of work activity in pregnancy on the risk of severe preeclampsia. Aust N Z J Obstet Gynaecol 1995; 35:380-5.

59. Campbell MK, Mottola MF. Recreational exercise and occupational activity during pregnancy and birth weight: A case-control study. Am J Obstet Gynecol 2001; 184:403-8.

60. Mendonça CP, Anjos LA. Aspectos das práticas alimentares e da atividade física como determinantes do crescimento do sobrepeso/obesidade no Brasil. Cad Saúde Pública 2004; 20:698-709.

Submitted on 14/Feb/2007

Final version resubmitted on 30/May/2007 Approved on 25/Jun/2007 